\title{
ISM Band Haberleşme Uygulamaları İçin Origami Anten Tasarımı
}

\author{
Alper Çalışkan ${ }^{1}$, Ahmet Kızılay², Mehmet Ali Belen ${ }^{3 *}$, Peyman Mahouti ${ }^{4}$ \\ ${ }^{1}$ Yıldız Teknik Üniversitesi, Elektrik Elektronik Fakültesi, Elektronik ve Haberleşme Mühendisliği Bölümü, 34300, İstanbul, Türkiye, (ORCID: 0000-0002-4608- \\ 8023) \\ ${ }^{2}$ Yıldız Teknik Üniversitesi, Elektrik Elektronik Fakültesi, Elektronik ve Haberleşme Mühendisliği Bölümü, 34300, İstanbul, Türkiye, (ORCID: 0000-0002-4099- \\ 8288) \\ ${ }^{3}$ Artvin Çoruh Üniversitesi, Mühendislik Fakültesi, Elektrik - Elektronik Mühendisliği Bölümü, 0800, Artvin, Türkiye, (ORCID: 0000-0001-5588-9407) \\ ${ }^{4}$ Arel Üniversitesi, Mühendislik-Mimarlık Fakültesi, Elektrik - Elektronik Mühendisliği Bölümü, 34300, İstanbul, Türkiye, (ORCID: 0000-0002-3351-4433)
}

(İlk Geliş Tarihi 1 Haziran 2019 ve Kabul Tarihi 28 Temmuz 2019)

(DOI: 10.31590/ejosat.573379)

ATIF/REFERENCE: Çalışkan, A., Kızılay, A., Belen, M. A. \& Mahouti, P. (2019). ISM Band Haberleşme Uygulamaları İçin Origami Anten Tasarımı. Avrupa Bilim ve Teknoloji Dergisi, (16), 785-791.

\section{$\ddot{O} \mathbf{z}$}

Son yıllarda, ebatları büyük antenlerin maliyetleri düşürmek adına minyatürleştirilmesi giderek artan bir ihtiyaç haline gelmiştir. Özellikle ticari ve askeri uygulamalar için küçük boyutlu, hafif ve geniş bantlı anten tasarımları önem kazanmıştır. Bu sebeple yeniden yapılandırılabilen antenler son yıllarda haberleşme alanındaki ihtiyaçlardan dolayı popüler hale gelmektedir. Yeniden yapılandırılabilen antenler çok fonksiyonlu ve çok bantlı çalışmalar gibi benzersiz avantajlara sahip olmasının yanı sıra, tasarımların minyatürleştirilmesi sayesinde ilgi çekmektedir. Boyut indirgeme amaçlı yeniden yapılandırılabilen anten tasarımında kullanılan tekniklerden biri de Origami katlama sanatıdır. Origami iki boyutlu ortamı katlama vasıtasıyla istenen belirli özelliklerde belirli bir biçime getirilmesi olarak tanımlanabilir. Origami antenler hacim veya alandan tasarruf sağlamaları yönünden oldukça kullanışlıdır. Bununla birlikte Origami şekillerinin, hâli hazırda kullanılan antenlerin yerlerine geçerek klasik anten sistemlerinde köklü yenilikler getirebileceği düşünülmektedir. $\mathrm{Bu}$ çalışma kapsamında küçük boyutlu, taşınabilir, katlanabilir, düşük maliyetli ve ayarlanabilir yapılı anten tasarım problemini aşmak için değişebilen katlamalı/Origami mikrodalga antenler incelenmiştir. Bu çalışma kapsamında ISM bant haberleşme sistemleri için loop ve helis anten modelleri incelenmiştir. Tasarımlarda CST Microwave Studio programı kullanılarak anten yapılarına ait bant genişliği, yansıma $S_{11}$, kazanç ve 1şıma örüntüsüne ek, antenin katlama-açılma durumuna göre tasarımlara ait performans değişimleri incelenmiştir. Bu çalışma kapsamında $2.4 \mathrm{GHz}$ frekans bandı uygulamaları için katlanabilir, yüksek kazançlı origami anten modelleri (Yagi-loop ve Helis) tasarımı yapılmıştır. Yapılan simülasyonlar sonucunda antenlerin geri-dönüş kaybı $\mathrm{S}_{11}-10 \mathrm{~dB}$ altında elde edilmiştir. Anten boyutları katlanmaları ile \%75-\%80 oranında küçülebilmektedir. Yapılan simülasyonlar sonucunda origami anten yapısı sayesinde ağırlık, taşınabilirlik, istifleme, maliyet, yüksek performans, huzme yönlendiriciliği açısından önemli derecede katkı sağlayabileceği görülmüştür. Önerilen anten yapısının belirtilen özeliklerinden dolayı birçok askeri ve ticari uygulamada avantaj sağlayacağı düşünülmektedir.

Anahtar Kelimeler: Origami anten, Boyut azaltma, Yeniden yapılandırılan anten, ISM band uygulamaları.

\section{Design of Origami Antenna for ISM Band Applications}

\begin{abstract}
In recent years, the miniaturization of large sized antennas had become a necessity for low cost designs. Reconfigurable antenna had become popular recently due to their ability of being multifunctional, multi band alongside of ability of miniaturization in designs. One
\end{abstract}

\footnotetext{
* Sorumlu Yazar: Artvin Çoruh Üniversitesi, Mühendislik Fakültesi, Elektrik - Elektronik Mühendisliği Bölümü, 0800, Artvin, Türkiye, ORCID: 00000001-5588-9407, mehmetalibelen@hotmail.com
} 
of the techniques of miniaturization for reconfigurable antenna is Origami folding. Origami antenna designs are efficient designs in means of reduced volume and size. Thus, it is possible to make great innovations in the currently used classical antenna designs with the usage of Origami designs. Herein, in order to achieve the challenging problem of, low size, mobile, foldable, low cost and reconfigurable antenna design Origami microwave antennas had been studied. İn this work, origami Loop and Helix antenna models for ISM band applications are investigated. The performance measures of Band-width, Return loss $\mathrm{S}_{11}$, Gain and radiation pattern of designs had been analysed using CST Microwave Studio. The performance measures of designs had been compared for the case of open and folded versions of antennas. Herein, design of high performance origami antennas (Yagi loop and helix) has been studied for applications at $2.4 \mathrm{GHz}$ frequency band. The return loss characteristic of the simulated models has achieved a $\mathrm{S}_{11}$ level of less than -10 $\mathrm{dB}$. With the folding ability of the antennas it is possible to reduce the total size of the antennas up to $75-80 \%$. From the simulated results it can be concluded that with the usage of origami it is possible to achieve designs with reduced weight, higher mobility, low cost, high performance and designs with reconfigurable pattern ability. Thus, it can be concluded that the proposed antennas can be taken as advantages design for many military or civil communication applications.

Keywords: Origami antenna, Size reduction, Reconfigurable antenna, ISM band applications.

\section{Giriş}

Origami iki boyutlu ortamı katlama vasıtasıyla istenen belirli özelliklerde belirli biçime getirmek olarak tanımlanabilir. En basit yaklaşımla, belirgin şekil derin simetride tek renkli kare parçanın sadece düz çizgide katlanması ile gerçekleştirilebilir; basit olmayan yaklaşımla ise, düz ya da kıvrımlı çizgiler boyunca kesmek ya da yarıklar açmak; çeşitli taslaklar ile (belirli özellikleri elde edebilmek amacıyla) çok renkli ortamları oluşturmak için kullanılır (Demaine ve ark., 2007). Özel katlama, yöntem ve paternler ile oluşturulan şekiller anten tasarımında boyut azaltma ve band genişliği artırma gibi performans parametrelerinde ciddi iyileştirmeler sağlayabilir. Origami katlama sanatı tekniklerini yumuşak ve/veya katlanabilir, mikrodalga çalışma frekanslarına uygun malzemelerde kullanılması ile yüksek performanslı düşük boyutlu anten tasarımları gerçekleştirilebilir. Origami tasarımında, origami şeklinin açılmış durumu iki boyutludur, bu nedenle katlama kalıbı ve origami döşemeleri iki boyutlu düşünmeyi gerektirir. Diğer bir ifadeyle origami tasarımı iki boyutlu tasarım vasıtasıyla elde edilen üç boyutlu bir tasarımdır. Üç boyutlu tasarım düşünsel olarak tüm şeklin göz önünde canlandırılması ve şeklin düşünsel olarak tüm yönlerde çevrilmesi yeteneğini ve ayrıca kütlenin etkisini, farklı malzemelerin tabiatını, uzay ve derinliğin baştan sona akışını keşfetme yeteneğini gerektirir. Bunun yanında hem iki boyutlu hem de üç boyutlu düşüncenin kaçınılmaz gerekliliği ise tasarımcının iki görevi eş zamanlı olarak yerine getirme durumunda olmasıdır: tasarımcılık ve mühendislik. Sadece tamamlanmış modelin görünümü değil, şeklin gerçeklenmesine nasıl devam edilebileceği ile ilgili stratejik kararlar da hesaba katılmalıdır. Bu nedenle, şüphesiz ki origami tasarımı oldukça zordur ve fikri mülkiyet gerektirir (Felton ve ark., 2014).

Bu çalışmada, küçük boyutlu, taşınabilir, katlanabilir, düşük maliyetli ve ayarlanabilir yapılı anten tasarım problemini aşmak için fraktal şekilli origami antenler incelenmiştir. Fraktal şekilli anten yapıları kullanılarak geleneksel bir anten modelinde (mikroşerit yama anten) gibi bir yapıda fraktal şekilli tasarımlar ile benzer performansı çok daha küçük bir alanda elde etmek mümkün olacaktır. Önemli olan yapıda kullanılacak olan fraktal şekilleri kuplaj gibi tahmini zor etkilerinin iyice hesaplanması ve ona göre modellenmeleridir. Günümüzde birçok 3 boyutlu elektromanyetik simulator programı ile bu tasarımlar ciddi anlamda kolaylaşmıştır. Origami fraktal yapılar mekanik düzenekler ile tasarım boyutu katlanma yöntemleri ile değiştirilerek antenin frekans bandı, ışıma paterni, kazanç değeri ve yansıma değerlerini değiştirerek farklı amaçlar için kullanılabilen yapılar oluşturulması mümkün olacaktır. Başka bir uygulama olarak kâğıt üzerine ideal bir helis anten modeli tasarımı yapıldıktan sonra yüksekliğin değişmesiyle antenin sarımlarının arasındaki mesafe düzgün bir şekilde azalıp arttırılabilmektedir. Bu tasarım ISM bant haberleşmesi ve GPS sistemlerinde yeni bir yaklaşım sağlamaktadır. Mesafeyi dönme açısına bağlı olarak ifade edersek, dönme açısının teorik sınır aralığı 30 ile 60 derece arasındadır. Ayrıca akordeon origami yapısı genişleyip çökebilme özelliklerine sahiptir. Bu yapıda yüksekliğe bağlı olarak origami antenin çalışma frekansı değiştirilebilmektedir. Katlanabilirlik özelliği genellikle konuşlandırma yapılan çeşitli uygulamalarda avantaj sağlamaktadır.

\section{Materyal ve Metot}

\subsection{Origami Elektroniği ve Origami Antenler}

Origami tekniği robotlar, enerji panelleri, teleskopik lensler arasında değişen alanlarda mühendisler tarafından ilgili görülmüştür (Felton ve ark., 2014). Çünkü anten uygulamalarında (Gerard ve ark., 2014; Yao ve ark., 2014), antenin boyutunun yapılandırılması veya performans arttırımını sağlamak için origami geometriler avantaj sağlamıştır. Ayrıca engellenmiş yeniden konfigüre edilebilir bir origami konik spiral anten tasarlanmış ve eksenel ışımasını iki durum için incelenmiştir (Liu ve ark., 2015). Şekil 1,'de verilen origami anten modelinde $2.07 \mathrm{GHz}, 3 \mathrm{GHz}$ ve $4.45 \mathrm{GHz}$ arasındaki çalışma frekansları için yeni bir origami quadrifilar sarmal anten (QHA) sunmuşlardır (Liu ve ark., 2015; Liu ve ark., 2015). Origami reflektör tüm çalışma frekansları için QHA kazancı artırmak için tasarlanmıştır. Yeniden yapılandırılabilir reflektör ile bu origami QHA tipik simüle gerçekleşen kazanç $2.07 \mathrm{GHz} 12.82 \mathrm{~dB}, 3 \mathrm{GHz}$ (15.13 dB), ve $4.45 \mathrm{GHz}$ 'de $15.13 \mathrm{~dB}$ bulunmaktadır. 


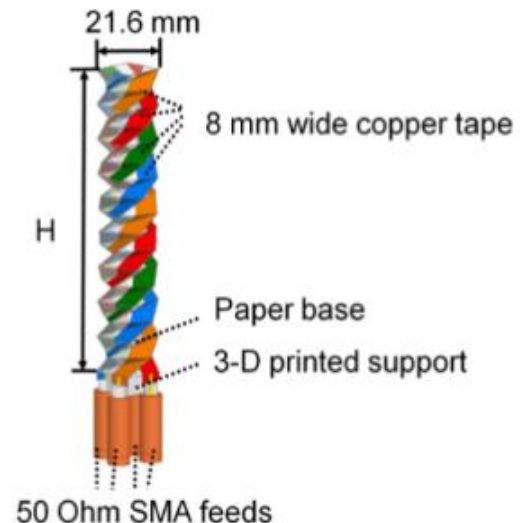

(a)

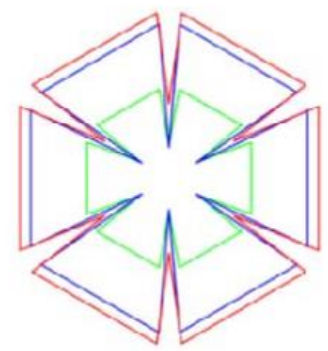

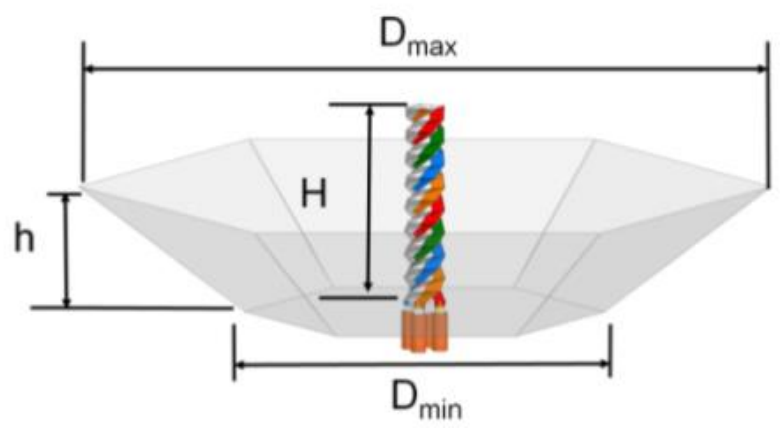

(b)

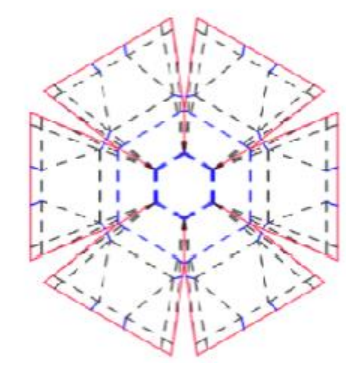

(c)

Şekil 1.QHA model origami anteni (Liu ve ark., 2015)

2-mod yeniden konfigüre origami Nojima anten şekil 2'te verilmiştir (Yao ve ark., 2015). Anten katlandığında, bu yön modunu oluşturmakta ve tamamen açılmış olduğunda, çok yönlü olmaktadır. Bu antenin operasyon frekansı katlanmış durumdaki $1.61 \mathrm{GHz}$ ve katlanmamış halde $0.66 \mathrm{GHz}$ dir.

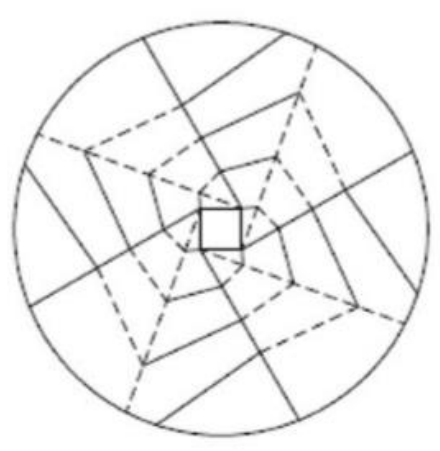

(a)

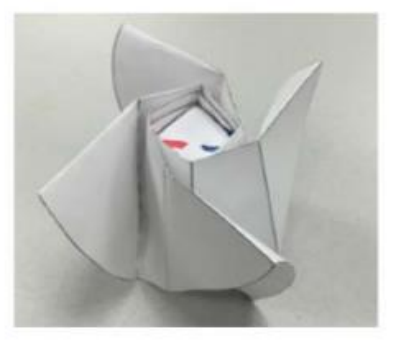

(b)

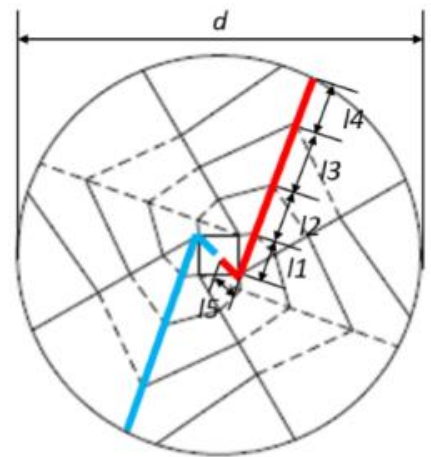

(c)

Şekil 2.Nojima model origami anteni

Origami anten yapıları ile RF hasatlayıcı devrelerde performans artırımı ve boyut küçültme amacıyla sistem tasarımı üzerine çalışılmıştır. Tasarımda tüm elektronik tasarım 3D yapı içinde yer almaktadır ve antenler 3D yapının yüzeylerinde bulunarak kablosuz sensör ağı düğümlerinde radyo frekansı (RF) enerjisi hasat uygulamaları için kullanılmıştır (FKimionis ve ark., 2015). [9]. Değiş̧imi sağlayan menteşeler çapraz bir kübik şekil için "origami" tarzında katlanarak mekanik destek sağlamaktadır.

\section{Araştırma Sonuçları ve Tartışma}

\subsection{Origami Anten Tasarımı ve Parametreleri}

İlk aşamada anten tasarımının performans parametreleri incelenmiştir. Bu parametreler; antenin geri donuş kaybı, ışıma örüntüsü, kazanç ve hüzme genişliği başlıkları altında toplanmıştır. Çalışma kapsamında literatürde yaygın olarak kullanılan Yagi-loop ve helis anten tipleri incelenmiştir. Bu antenlerin seçilme nedeni ISM Bant haberleşme sistemlerinde yaygın olarak kullanılmalarıdır. Origami 
tekniği ile bu antenler yeniden modellenerek, $2.4 \mathrm{GHz}$ ISM bandı uygulamaları için portatif anten modelleri oluşturulmuştur. Tasarımlarda origami yapısı sayesinde katlama-açılma yapısı ile anten boyutunu minimuma indirilmiştir.

Yagi-loop anten geometrik modeli ve tasarım parametreleri Şekil 3' ve tablo 1'de verilmiştir. Katlama-açılma yapısı ile anten boyutu $24 \mathrm{~mm}$ ile $120 \mathrm{~mm}$ arasında değişmektedir. Katlama-açılma özelliği sayesinde anten depolama hacmi \%80 oranında küçültülmüştür. Böylelikle origami anten yapısı taşınabilirlik, istifleme ve maliyet açısından önemli fayda sağlamaktadır.

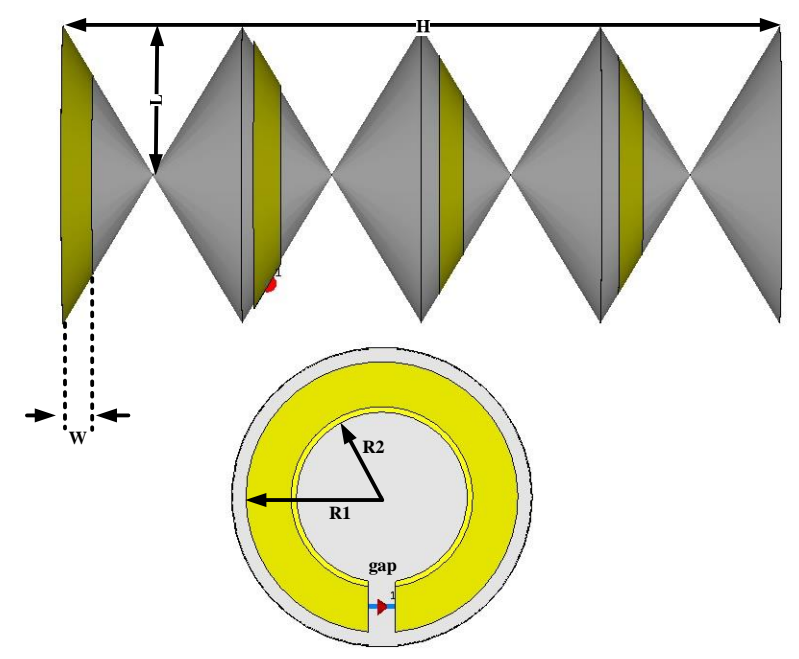

(a)

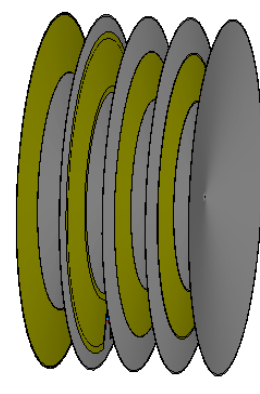

(b)

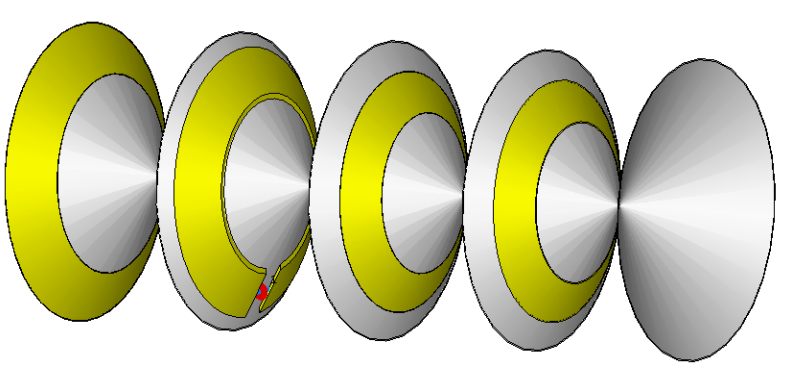

(c)

Şekil 2.Origami yagi-loop anten (a) Parametrik gösterimi, (b) Kapalı durumda 24mm, (c) Açık durumda 120mm

Tablo 1. Origami Yagi Loop Anten Parametre Listesi ( $\mathrm{mm}$ )

\begin{tabular}{|c|c|c|c|}
\hline $\mathbf{W}$ & 5 & $\mathbf{R 1}$ & 22.6 \\
\hline $\mathbf{L}$ & 25 & $\mathbf{R 2}$ & 15.1 \\
\hline $\mathbf{H}$ & 130 & gap & 4.4 \\
\hline
\end{tabular}

Şekil 4’te tasarlanan antenin 3-boyutlu 1şıma örüntüsü verilmiştir. Işıma şekillerinden görüleceği üzere anten 1şıması yönlüdür ve katlama /açılma özelliği ile kazanç $2 \mathrm{~dB}$ artmıştır. 


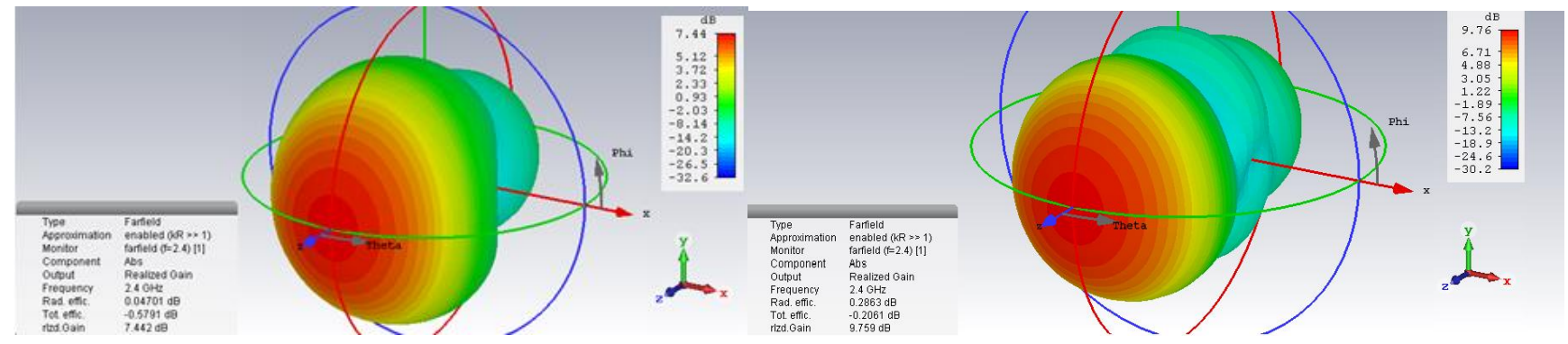

(a)

(b)

Şekil 4. Origami yagi anten ışıma örüntüsü (a) Katlı (24mm) (b) Açık (120mm)

Şekil 5'da tasarlanan birim anten modelinin $\mathrm{S}_{11}$, 1şıma örüntüsü verilmiştir. Çalışma frekansı $2.4 \mathrm{GHz}$ ile de $\mathrm{S}_{11}$ değeri -10dB altında, katlı ve açık durumda $7 \mathrm{~dB}$ ile $9 \mathrm{~dB}$ olarak elde edilmiştir.

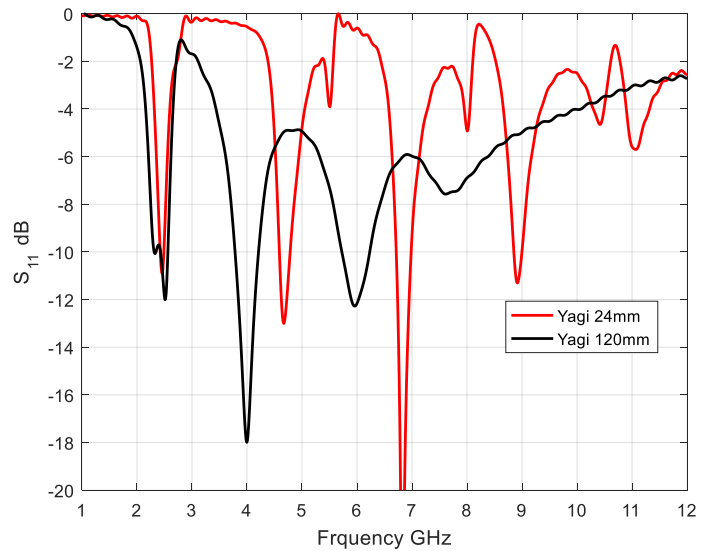

(a)

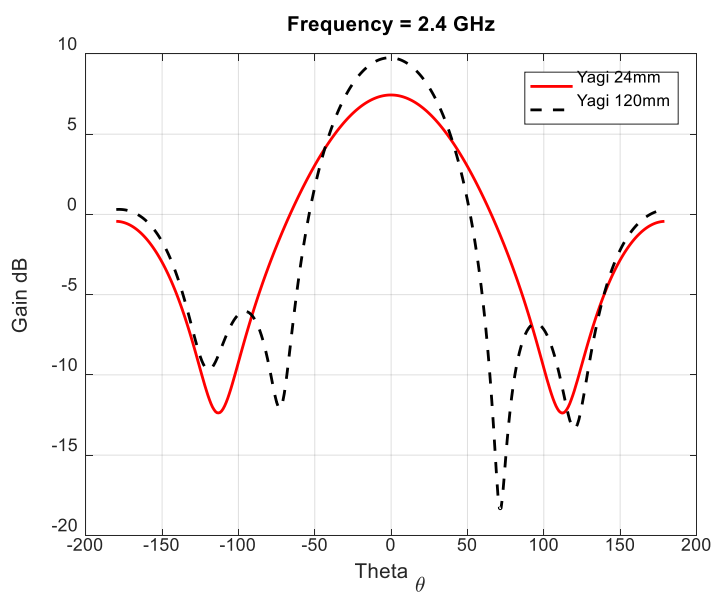

(b)

Şekil 5.Origami yagi-loop anten açık durumda (a) $S_{11}$ (b) Işıma Paterni

İkinci çalışma olarak ise helis antenin origami tekniği ile modellenmesi ele alınmıştır. Helis Anten geometrik modeli ve tasarım parametreleri Şekil 6' ve Tablo 2'de verilmiştir. Katlama-açılma yapısı ile anten boyutu 24mm ile 96mm arasında değişmektedir. Katlama-açılma özelliği ile anten depolama hacmi \%25'e indirilmektedir.

Tablo 2. Origami Helis Anten Parametre Listesi (mm)

\begin{tabular}{|c|c|c|c|}
\hline $\mathbf{H}$ & 120 & $\mathbf{L 1}$ & 100 \\
\hline $\mathbf{H 1}$ & 30 & $\mathbf{L 2}$ & 50 \\
\hline $\mathbf{H 2}$ & 5.5 & $\mathbf{W 1}$ & 100 \\
\hline $\mathbf{H 3}$ & 15 & $\mathbf{W} 2$ & 67 \\
\hline $\mathbf{H 4}$ & 2.12 & $\mathbf{R}$ & 30 \\
\hline
\end{tabular}




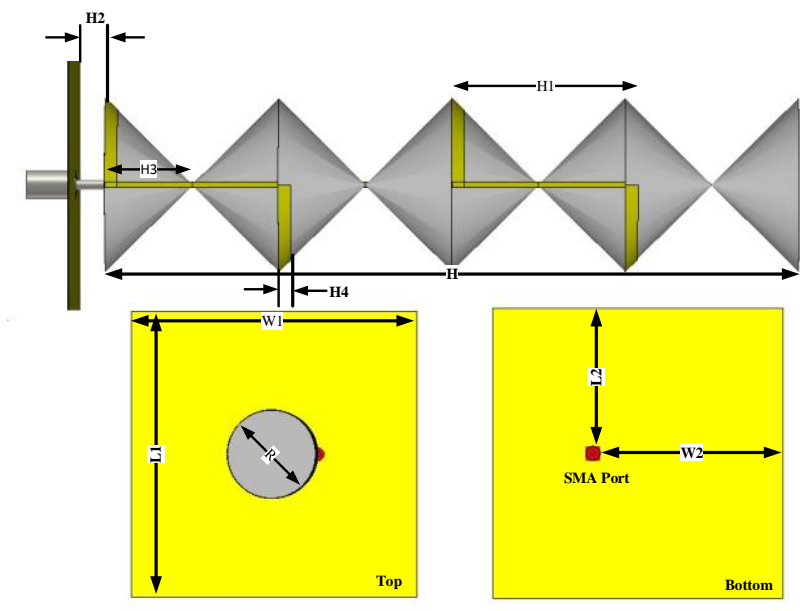

(a)

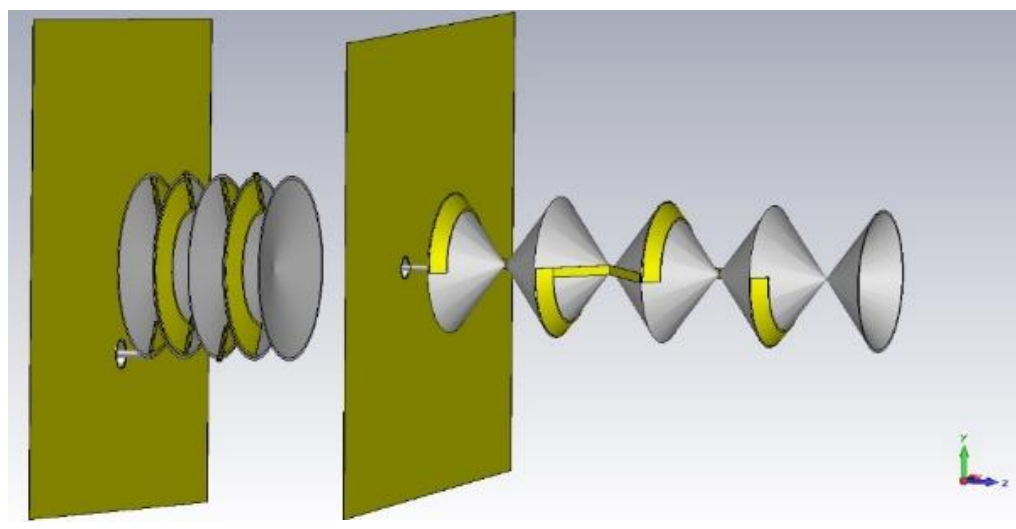

(b)

(c)

Şekil 6.Origami helis anten (a) Parametrik gösterimi, (b) Kapalı durumda 24mm (c) Açık durumda 96mm

Şekil 7'de tasarlanan helis antenin 3-boyutlu 1şıma örüntüsü verilmiştir. Işıma şekillerinden görüleceği üzere anten 1şıması yönlüdür ve katlama-açılma özelliği ile kazanç $4 \mathrm{~dB}$ artmıştır.

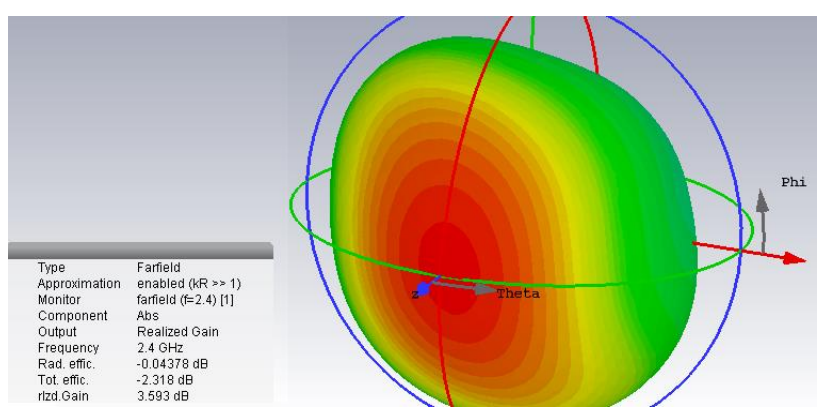

(a)

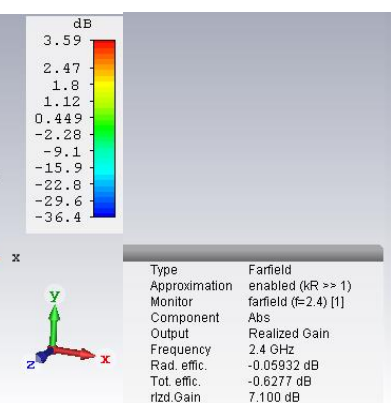

$7.100 \mathrm{~dB}$

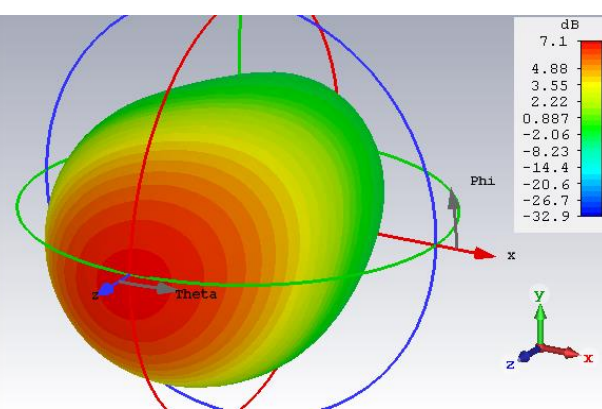

(b)

Şekil 7.Origami helis anten kazancı (a) $24 \mathrm{~mm}$ (b) $96 \mathrm{mmKazanç}$

Şekil 8'da tasarlanan birim anten modelinin $\mathrm{S}_{11}$, 1şıma paterni verilmiştir. Çalışma frekansı $2.4 \mathrm{GHz}$ ile de $\mathrm{S}_{11}$ değeri $-10 \mathrm{~dB}$ altında, katlı ve açık durumda kazanç değeri $3 \mathrm{~dB}$ ile $7 \mathrm{~dB}$ arasında değişmektedir. 


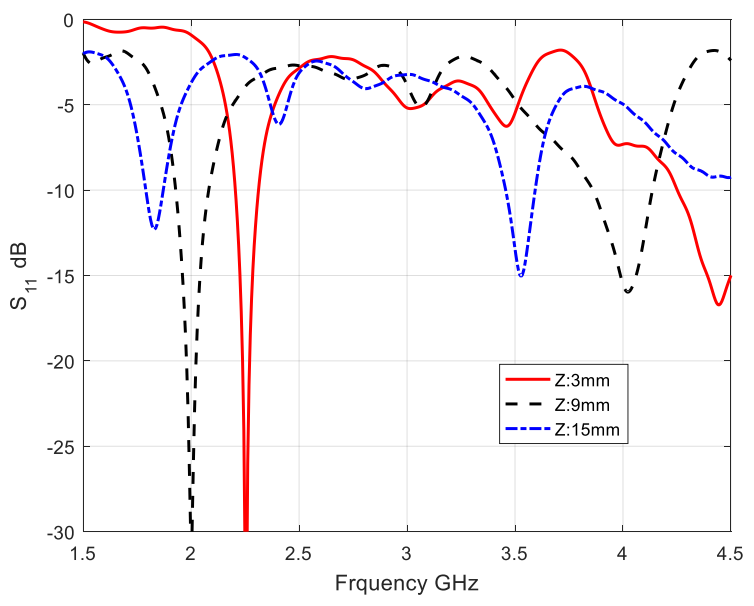

(a)

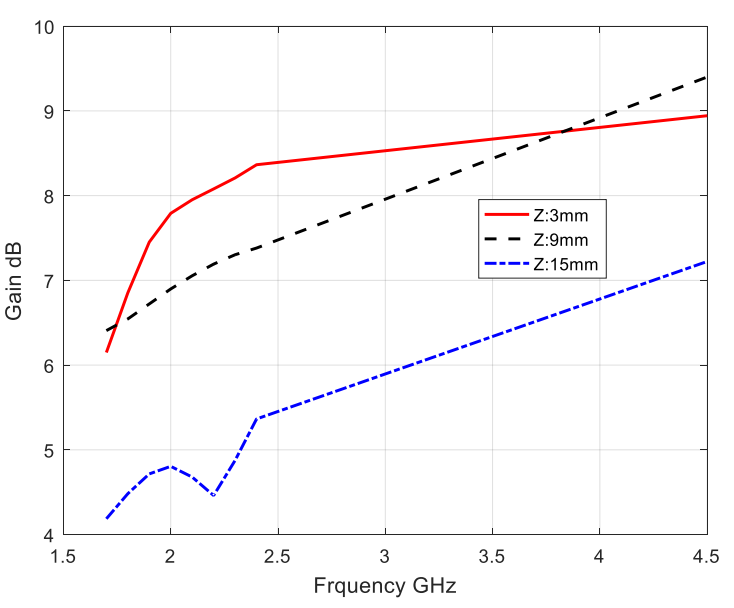

(b)

Şekil 8. Origami helis antene ait benzetim sonuçları (a) $S_{11}$, (b) Kazanç

2.4GHz ISM bandı haberleşme uygulamaları için geri dönüş kaybı $\mathrm{S}_{11}-10 \mathrm{~dB}$ altında elde edilmiştir. Anten boyutları \%75-\%80 arasında katlanması sayesinde ağırlık, taşınabilirlik, istifleme, maliyet, performans, hüzme yönlendiriciliği açısından önemli derecede etki sağlayabilecektir. Katlama ve açılma durumlarında anten kazancının değiştiği gözlenmiş ve anten modeline göre $2 \mathrm{~dB}$ ile $4 \mathrm{~dB}$ daha fazla kazanç elde edilmiştir.

\section{Sonuç}

Ticari ve askeri uygulamalar için küçük boyutlu, hafif ve geniş bantlı anten tasarımları önem kazanmıştır. Bu sebeple origami antenler son yıllarda haberleşme alanındaki ihtiyaçlardan dolayı popüler hale gelmektedir. Bu çalışma kapsamında $2.4 \mathrm{GHz}$ frekans bandı uygulamaları için katlanabilir, yüksek kazançlı origami anten modelleri (Yagi-loop ve Helis) tasarımı yapılmıştır. Yapılan simülasyonlar sonucunda antenlerin geri-dönüş kayb1 $\mathrm{S}_{11}-10 \mathrm{~dB}$ altında elde edilmiştir. Anten boyutları katlanmaları ile \% 75 -\%80 oranında küçülebilmektedir. Bu sayede taşınabilirlik, istifleme ve maliyet açısından önemli derecede fayda sağlanacaktır. Bu nedenle antenin katlanma ve açılma kapasitesine sahip olması birçok askeri ve ticari uygulamada avantaj sağlamaktadır. Bu tip antenler kablosuz haberleşmede, medikal cihazlarda, askeri araçlara monte edilen radar sistemlerinde ve uzay platformlarında kullanılabilme potansiyeline sahiptir.

\section{Kaynakça}

Demaine, E.D., O’Rourke J. (2007). Geometric Folding Algorithms: Linkages, Origami, Polyhedra. Cambridge University Press: New York

Gerard J. H., Ying L., Jan G., Gianluca L., Michael D. D. (2014). Self-Folding Origami Microstrip Antennas. IEEE Trans. Antennas \& Propagation, 62(10), 5416-5419. doi:10.1109/TAP.2014.2346188

Kimionis J., Georgiadis A., Isakov M., Qi H. J., Tentzeris, M. M. (2015). 3D/inkjet-printed origami antennas for multi-direction RF harvesting. 2015 IEEE MTT-S International Microwave Symposium, Phoenix, AZ, 1-4. doi: 10.1109/MWSYM.2015.7166878

Felton S., Tolley M., Demaine E., Rus D., Wood R. (2014). A method for building self-folding machines. Science, 345(6197), 644-646. doi: $10.1126 /$ science. 1252610

Yao S., Liu X., Georgakopoulos S. V., Tentzeris M. M. (2014). A novel reconfigurable origami spring antenna. 2014 IEEE Antennas and Propagation Society International Symposium (APSURSI). Memphis, TN, 374-375. doi: 10.1109/APS.2014.6904519

Yao S., Liu X., Georgakopoulos S. V. (2015). A mode reconfigurable Nojima origami antenna. 2015 IEEE International Symposium on Antennas and Propagation \& USNC/URSI National Radio Science Meeting. Vancouver, BC, 2237-2238. doi: 10.1109/APS.2015.7305507

Liu X., Yao S., Georgakopoulos S. V. (2015). Reconfigurable origami equiangular conical spiral antenna. 2015 IEEE International Symposium on Antennas and Propagation \& USNC/URSI National Radio Science Meeting, Vancouver, BC, 2263-2264. doi: 10.1109/APS.2015.7305520

Liu X., Yao S., Georgakopoulos S. V. (2015). Frequency reconfigurable origami quadrifilar helical antenna with reconfigurable reflector 2015 IEEE International Symposium on Antennas and Propagation \& USNC/URSI National Radio Science Meeting, Vancouver, BC, 2217-2218. doi: 10.1109/APS.2015.7305497

Liu X., Georgakopoulos S.V., Tentzeris M. (2015). A novel mode and frequency reconfigurable origami quadrifilar helical antenna. 2015 IEEE 16th Annual Wireless and Microwave Technology Conference (WAMICON), Cocoa Beach, FL, 1-3. doi: 10.1109/WAMICON.2015.7120416 